Article

\title{
Entropy Production Rate of a One-Dimensional Alpha-Fractional Diffusion Process
}

\author{
Yuri Luchko
}

Department of Mathematics, Physics, and Chemistry, Beuth Technical University of Applied Sciences, Luxemburger Str. 10, 13353 Berlin, Germany; luchko@beuth-hochschule.de

Academic Editor: Hans J. Haubold

Received: 30 December 2015; Accepted: 2 February 2016; Published: 5 February 2016

\begin{abstract}
In this paper, the one-dimensional $\alpha$-fractional diffusion equation is revisited. This equation is a particular case of the time- and space-fractional diffusion equation with the quotient of the orders of the time- and space-fractional derivatives equal to one-half. First, some integral representations of its fundamental solution including the Mellin-Barnes integral representation are derived. Then a series representation and asymptotics of the fundamental solution are discussed. The fundamental solution is interpreted as a probability density function and its entropy in the Shannon sense is calculated. The entropy production rate of the stochastic process governed by the $\alpha$-fractional diffusion equation is shown to be equal to one of the conventional diffusion equation.
\end{abstract}

Keywords: Caputo fractional derivative; Riesz fractional derivative; $\alpha$-fractional diffusion equation; Mellin transform; fundamental solution; Mellin-Barnes integrals; entropy; entropy production rate

MSC: 26A33; 35C05; 35E05; 35L05; 45K05; 60E99

\section{Introduction}

In the literature, many different kinds of the time-, space, and time- and space-fractional diffusion equations have been already introduced and analyzed. The fractional derivatives contained in the fractional diffusion equations are defined in the Riemann-Liouville, Weyl, Caputo, Riez, or Riesz-Feller sense to mention only some of the most used types of the fractional derivatives. From the mathematical viewpoint, all of these fractional diffusion equations can be seen as generalizations of the conventional diffusion equation and thus are worth to be investigated. On the other hand, it is not clear at all, what kinds of the fractional diffusion equations could/should be employed as mathematical models, say, for describing the phenomena of the anomalous diffusion (see, e.g., the recent survey paper [1] for about three hundred references to the relevant works). Usually, the anomalous diffusion processes are defined as those that do not longer follow the Gaussian statistics on the long time intervals. Especially, the linear time dependence of the mean squared displacement of the diffusing particles does not hold any more and has to be either replaced with a different (mainly power-law) dependence or the mean squared displacement does not exist at all. As a rule, the stochastic processes governed by different kinds of the fractional diffusion equations are non Gaussian and thus can be seen as potential candidates for the role of mathematical models for anomalous diffusion processes.

One more important characteristic of the diffusion processes is their entropy and the entropy production rate. The concept of entropy was first introduced in the macroscopic thermodynamics and then extended for description of some phenomena in statistical mechanics, information theory, ergodic theory of dynamical systems, etc. Historically, many definitions of entropy were proposed and applied in different knowledge areas. In this paper, we employ the statistical concept of entropy that goes back to Shannon and was introduced by him in the theory of communication and transmission of information (see [2]). The entropy of the processes governed by the time- and space-fractional diffusion 
equations has been discussed in [3-6], respectively. It is worth mentioning that according to [3,5] the entropy production rates for the time- and the space-fractional diffusion equations depend on the derivative order $\alpha$ of the time- or space-fractional derivative, respectively, and increase with increasing of $\alpha$ from 1 (diffusion) to 2 (wave propagation) that results in the so called entropy production paradox. In [7], entropy behavior of solutions to the one-dimensional neutral-fractional equation that contains fractional derivatives of the same order $\alpha, 1 \leq \alpha \leq 2$ both in space and in time, has been considered. It was shown in [7] that the entropy production rate of solutions to the neutral-fractional equation does not depend on the equation order $\alpha$ and is twice as much as the entropy production rate of solutions to the conventional diffusion equation. In this paper, we show that the entropy production rate of the fundamental solution to the $\alpha$-fractional diffusion equation is exactly the same as in the case of the conventional diffusion equation. Thus the $\alpha$-fractional diffusion equation combines the properties of the anomalous diffusion (the mean squared displacement of the diffusing particles does not exists) and of the conventional diffusion (the same entropy production rate) and could be considered to be a kind of a "natural fractionalization" of the diffusion equation. In this paper we restrict ourselves to the classical Shannon entropy, other kinds of generalized entropies (see e.g., [8-10] and the references therein) will be considered elsewhere.

From the mathematical viewpoint, the $\alpha$-fractional diffusion equation is a particular case of the one-dimensional space-time fractional diffusion equation that has been considered in [11] in detail. The equation studied in [11] contains the Riesz-Feller derivative of order $\alpha \in(0,2]$ and skewness $\theta$ and the Caputo fractional derivative of order $\beta \in(0,2]$. In particular, it was shown in [11] that the fundamental solution to the space-time fractional diffusion equation can be interpreted as a spatial probability density function evolving in time if $\{0<\alpha \leq 2\} \cap\{0<\beta \leq 1\}$ or if $\{1<\beta \leq \alpha \leq 2\}$. The $\alpha$-fractional diffusion equation we deal with in this paper corresponds to the case $\alpha=2 \beta, 0 \leq \beta \leq 1, \theta=0$ in the space-time fractional diffusion equation considered in [11] and possesses some remarkable properties that do not hold true for solutions of the general equation.

The rest of the paper is organized as follows. In the 2nd section, the basic definitions, problem formulation, and some analytical results for the initial-value problems for the one-dimensional $\alpha$-fractional equation are presented. Among other things, the Mellin-Barnes integral representation of the fundamental solution as well as its series representation and asymptotics are given. The last section is devoted to a probabilistic interpretation of the fundamental solution to the one-dimensional $\alpha$-fractional diffusion equation. In particular, the Shannon entropy and the entropy production rate are calculated. The entropy production rate of the stochastic process governed by the $\alpha$-fractional diffusion equation is shown to be independent on the equation order $\alpha$ and is exactly the same as the entropy production rate of the conventional diffusion process.

\section{Alpha-Fractional Diffusion Equation}

\subsection{Problem Formulation}

In this paper, we deal with the one-dimensional $\alpha$-fractional diffusion equation in the form

$$
D_{t}^{\alpha} u(x, t)=-(-\Delta)^{\alpha} u(x, t), x \in \mathbb{R}, t \in \mathbb{R}_{+}, 0<\alpha \leq 1
$$

In the Equation (1), $D_{t}^{\alpha}$ is the Caputo time-fractional derivative of order $\alpha$ defined by

$$
\left(D^{\alpha} f\right)(t)=\left(I^{n-\alpha} f^{(n)}\right)(t), n-1<\alpha \leq n, n \in \mathbb{N},
$$

$I^{\alpha}, \alpha \geq 0$ being the Riemann-Liouville fractional integral

$$
\left(I^{\alpha} f\right)(t)=\left\{\begin{array}{l}
\frac{1}{\Gamma(\alpha)} \int_{0}^{t}(t-\tau)^{\alpha-1} f(\tau) d \tau, \alpha>0 \\
f(t), \alpha=0
\end{array}\right.
$$


and $\Gamma$ the Euler gamma function. For $\alpha=n, n \in N$, the Caputo fractional derivative coincides by definition with the derivative of order $n$.

For a sufficiently well-behaved function $f$, the Riesz fractional derivative $-(-\Delta)^{\alpha}$ is defined as a pseudo-differential operator with the symbol $-|\kappa|^{2 \alpha}$ (see e.g., [11-14]):

$$
\left(\mathcal{F}-(-\Delta)^{\alpha} f\right)(\kappa)=-|\kappa|^{2 \alpha}(\mathcal{F} f)(\kappa),
$$

$\mathcal{F}$ being the Fourier transform of a function $f$ defined by the formula

$$
(\mathcal{F} f)(\kappa)=\hat{f}(\kappa)=\int_{-\infty}^{+\infty} f(x) e^{i x \kappa} d x
$$

The Riesz fractional derivative (3) can be represented as a hypersingular integral under the condition $0<\alpha<1$ (see [14] for the case $\alpha \neq \frac{1}{2}$ and [12] for the general case)

$$
-(-\Delta)^{\alpha} f(x)=\frac{1}{\pi} \Gamma(1+2 \alpha) \sin (\alpha \pi) \int_{0}^{\infty} \frac{f(x+\xi)-2 f(x)+f(x-\xi)}{\xi^{2 \alpha+1}} d \xi .
$$

For $\alpha=\frac{1}{2}$, the relation (4) can be interpreted in terms of the Hilbert transform

$$
-(-\Delta)^{\frac{1}{2}} f(x)=-\frac{1}{\pi} \frac{d}{d x} \int_{-\infty}^{+\infty} \frac{f(\xi)}{x-\xi} d \xi
$$

where the integral is understood in the sense of the Cauchy principal value as first noted in [15] and then revisited and stated more precisely in $[12,16]$.

Let us note that the Riesz fractional derivative is a symmetric operator with respect to the space variable $x$. Because of the relation $-|\kappa|^{2 \alpha}=-\left(\kappa^{2}\right)^{\alpha}$ it can be formally interpreted as

$$
\frac{d^{2 \alpha}}{d|x|^{2 \alpha}}=-\left(-\frac{d^{2}}{d x^{2}}\right)^{\alpha}
$$

i.e., as a power of the self-adjoint and positive definite operator $-\frac{d^{2}}{d x^{2}}$.

For $\alpha=1$, Equation (1) is reduced to the one-dimensional diffusion equation. In what follows, we focus on the case $0 \leq \alpha<1$ because the case $\alpha=1$ (diffusion equation) is well studied in the literature.

In the rest of the paper, we consider the initial-value problem

$$
u(x, 0)=\varphi(x), \quad x \in \mathbb{R}
$$

for the Equation (1). In doing so, we are mostly interested in behavior and properties of the fundamental solution (Green function) $G_{\alpha}=G_{\alpha}(x, t)$ of the Equation (1), i.e., in its solution with the initial condition $\varphi(x)=\delta(x), \delta$ being the Dirac delta function.

\subsection{Fundamental Solution of the Alpha-Fractional Diffusion Equation}

In this subsection, we follow the derivations presented in [11] for the more general case of the one-dimensional time-space fractional diffusion equation with some minor modifications. To determine the fundamental solution $G_{\alpha}$ let us apply the Fourier transform to the Equation (1) and to the initial conditions (5) with $\varphi(x)=\delta(x)$. Using definition of the Riesz fractional derivative, for the Fourier transform $\hat{G}_{\alpha}$ we get then the initial-value problem

$$
\hat{G}(\kappa, 0)=1
$$


for the fractional differential equation

$$
\left(D^{\alpha} \hat{G}_{\alpha}\right)(t)+|\kappa|^{2 \alpha} \hat{G}_{\alpha}(\kappa, t)=0 .
$$

The unique solution of (6), (7) is given by the expression (see e.g., $[17,18]$ )

$$
\hat{G}_{\alpha}(\kappa, t)=E_{\alpha}\left(-|\kappa|^{2 \alpha} t^{\alpha}\right)
$$

in terms of the Mittag-Leffler function $E_{\alpha}$ that is defined as an convergent power series

$$
E_{\alpha}(z)=\sum_{k=0}^{\infty} \frac{z^{k}}{\Gamma(1+\alpha k)}, \alpha>0
$$

As follows from the well-known asymptotic formula

$$
E_{\alpha}(-x)=-\sum_{k=1}^{m} \frac{(-x)^{-k}}{\Gamma(1-\alpha k)}+O\left(x^{-1-m}\right), m \in N, x \rightarrow+\infty, 0<\alpha<2,
$$

the Fourier transform $\hat{G}_{\alpha}$ belongs to $L_{1}(\mathbb{R})$ with respect to $\kappa$ under the condition $\alpha>\frac{1}{2}$. In the further discussions we suppose that this conditions holds true. Then we can apply the inverse Fourier transform and get the representation

$$
G_{\alpha}(x, t)=\frac{1}{2 \pi} \int_{-\infty}^{+\infty} e^{-i \kappa x} E_{\alpha}\left(-|\kappa|^{2 \alpha} t^{\alpha}\right) d \kappa, x \in \mathbb{R}, t>0
$$

that can be rewritten as the cos-Fourier transform:

$$
G_{\alpha}(x, t)=\frac{1}{\pi} \int_{0}^{\infty} \cos (\kappa x) E_{\alpha}\left(-\kappa^{2 \alpha} t^{\alpha}\right) d \kappa, x \in \mathbb{R}, t>0 .
$$

Now we are going to apply the technique of the Mellin integral transform to deduce the Mellin-Barnes representation of the fundamental solution. For the reader's convenience, some basic elements of the Mellin integral transform theory are presented below.

The Mellin integral transform of a function $f$ is defined by the formula

$$
f^{*}(s)=(\mathcal{M} f)(s)=\int_{0}^{\infty} f(t) t^{s-1} d t, \gamma_{1}<\Re(s)<\gamma_{2},
$$

and the inverse Mellin integral transform by the formula

$$
f(t)=\left(\mathcal{M}^{-1} f^{*}\right)(t)=\frac{1}{2 \pi i} \int_{\gamma-i \infty}^{\gamma+i \infty} f^{*}(s) t^{-s} d s, t>0, \Re(s)=\gamma, \gamma_{1}<\gamma<\gamma_{2} .
$$

The Mellin integral transform and its inversion exist under the following sufficient conditions (see e.g., [19]): Let $f \in L^{c}(\epsilon, E), 0<\epsilon<E<\infty$ be a function continuous on the intervals $(0, \epsilon]$ and $[E, \infty)$, and let $|f(r)| \leq M r^{-\gamma_{1}}$ for $0<r<\epsilon,|f(r)| \leq M r^{-\gamma_{2}}$ for $r>E$, where $M$ is a constant. If $\gamma_{1}<\gamma_{2}$, then the Mellin transform (12) of the function $f$ exists and is analytic in the vertical strip $\gamma_{1}<\gamma=\Re(s)<\gamma_{2}$.

If $f$ is piecewise differentiable and $f(r) r^{\gamma-1} \in L^{c}(0, \infty)$, then the Formula (13) holds true in all points where $f$ is continuous. The integral in (13) must be understood in the sense of the Cauchy principal value. 
Let us denote by $\stackrel{\mathcal{M}}{\leftrightarrow}$ the juxtaposition of a function $f$ with its Mellin transform $f^{*}$. With this notation the convolution theorem for the Mellin convolution reads as follows:

$$
\int_{0}^{\infty} g(\kappa) f(y / \kappa) \frac{d \kappa}{\kappa} \stackrel{\mathcal{M}}{\leftrightarrow} g^{*}(s) f^{*}(s) .
$$

Now let us return back to the integral representation (11) of the fundamental solution $G_{\alpha}$ and consider the cases a) $x=0$ and b) $x \neq 0$.

(a) For $x=0$, the integral at the right-hand side of (11) can be interpreted as the Mellin integral transform of the Mittag-Leffler function at the point $s=\frac{1}{2 \alpha}$. It converges under the condition $\alpha>\frac{1}{2}$ and its value is given by the formula (see e.g., [19])

$$
\begin{aligned}
& \frac{1}{\pi} \int_{0}^{\infty} E_{\alpha}\left(-\kappa^{2 \alpha} t^{\alpha}\right) d \kappa=\frac{1}{2 \pi \alpha \sqrt{t}} \int_{0}^{\infty} E_{\alpha}(-u) u^{\frac{1}{2 \alpha}-1} d u \\
= & \frac{1}{2 \pi \alpha \sqrt{t}} \frac{\Gamma\left(\frac{1}{2 \alpha}\right) \Gamma\left(1-\frac{1}{2 \alpha}\right)}{\Gamma\left(1-\alpha \frac{1}{2 \alpha}\right)}=\frac{1}{\alpha \sqrt{4 \pi t} \sin \left(\frac{\pi}{2 \alpha}\right)}, t>0 .
\end{aligned}
$$

For $\alpha=1$, the Green function $G_{\alpha}$ is a time-dependent Gaussian distribution $G_{1}(x, t)=\frac{1}{\sqrt{4 \pi t}} \exp \left(-\frac{x^{2}}{4 t}\right)$ and thus $G_{1}(0, t)=\frac{1}{\sqrt{4 \pi t}}$ that is in accordance with the above formula for the value of $G_{\alpha}(0, t)$.

(b) In the case $x \neq 0$, we recognize that the integral at the right-hand side of (11) can be interpreted as the Mellin convolution of the functions

$$
g(\kappa)=E_{\alpha}\left(-\kappa^{2 \alpha} t^{\alpha}\right) \text { and } f(\kappa)=\frac{1}{\pi|x| \kappa} \cos \left(\frac{1}{\kappa}\right)
$$

at the point $y=\frac{1}{|x|}$.

Using the known Mellin integral transforms of the cos-function and the Mittag-Leffler function as well as some elementary properties of the Mellin integral transform (see $[19,20])$ we get the formulas:

$$
\begin{gathered}
g^{*}(s)=\frac{1}{2 \alpha t^{\frac{s}{2}}} \frac{\Gamma\left(\frac{s}{2 \alpha}\right) \Gamma\left(1-\frac{s}{2 \alpha}\right)}{\Gamma\left(1-\frac{s}{2}\right)}, 0<\Re(s)<2 \alpha, \\
f^{*}(s)=\frac{1}{\sqrt{\pi}|x| 2^{s}} \frac{\Gamma\left(\frac{1}{2}-\frac{s}{2}\right)}{\Gamma\left(\frac{s}{2}\right)}, 0<\Re(s)<1 .
\end{gathered}
$$

These formulas together with the convolution theorem and the inverse Mellin integral transform lead to the following Mellin-Barnes representation of the fundamental solution $G_{\alpha}$ :

$$
G_{\alpha}(x, t)=\frac{1}{2 \alpha \sqrt{\pi}|x|} \frac{1}{2 \pi i} \int_{\gamma-i \infty}^{\gamma+i \infty} \frac{\Gamma\left(\frac{1}{2}-\frac{s}{2}\right) \Gamma\left(\frac{s}{2 \alpha}\right) \Gamma\left(1-\frac{s}{2 \alpha}\right)}{\Gamma\left(\frac{s}{2}\right) \Gamma\left(1-\frac{s}{2}\right)}\left(\frac{2 \sqrt{t}}{|x|}\right)^{-s} d s, 0<\gamma<\min \{1,2 \alpha\} .
$$

The linear variables substitution $s \rightarrow 2 s$ in the integral at the right-hand side of (15) leads to the representation

$$
G_{\alpha}(x, t)=\frac{1}{\alpha \sqrt{\pi}|x|} \frac{1}{2 \pi i} \int_{\gamma-i \infty}^{\gamma+i \infty} \frac{\Gamma\left(\frac{1}{2}-s\right) \Gamma\left(\frac{s}{\alpha}\right) \Gamma\left(1-\frac{s}{\alpha}\right)}{\Gamma(s) \Gamma(1-s)}\left(\frac{4 t}{x^{2}}\right)^{-s} d s, 0<\gamma<\min \{1 / 2, \alpha\}
$$

and then to the formula

$$
G_{\alpha}(x, t)=\frac{1}{\alpha \sqrt{\pi}|x|} \frac{1}{2 \pi i} \int_{\gamma-i \infty}^{\gamma+i \infty} \Gamma\left(\frac{1}{2}-s\right) \frac{\sin (\pi s)}{\sin (\pi s / \alpha)}\left(\frac{4 t}{x^{2}}\right)^{-s} d s, 0<\gamma<\min \{1 / 2, \alpha\}
$$


by using the reflection formulas for the gamma function.

In the next section, we need one more Mellin-Barnes integral representation of the fundamental solution $G_{\alpha}$ that is obtained from the Formula (16) using the linear variables substitution $s \rightarrow-s$ :

$$
G_{\alpha}(x, t)=\frac{1}{\alpha \sqrt{\pi}|x|} \frac{1}{2 \pi i} \int_{\gamma-i \infty}^{\gamma+i \infty} \frac{\Gamma\left(\frac{1}{2}+s\right) \Gamma\left(-\frac{s}{\alpha}\right) \Gamma\left(1+\frac{s}{\alpha}\right)}{\Gamma(-s) \Gamma(1+s)}\left(\frac{x^{2}}{4 t}\right)^{-s} d s,-\min \{1 / 2, \alpha\}<\gamma<0 .
$$

From Equation (18), a useful representation

$$
G_{\alpha}(x, t)=\frac{1}{|x|} L_{\alpha}\left(\frac{x^{2}}{4 t}\right), x \neq 0, t>0
$$

of the fundamental solution $G_{\alpha}$ in terms of an auxiliary function $L_{\alpha}$ defined by its Mellin-Barnes representation

$$
L_{\alpha}(\tau)=\frac{1}{\alpha \sqrt{\pi}} \frac{1}{2 \pi i} \int_{\gamma-i \infty}^{\gamma+i \infty} \frac{\Gamma\left(\frac{1}{2}+s\right) \Gamma\left(-\frac{s}{\alpha}\right) \Gamma\left(1+\frac{s}{\alpha}\right)}{\Gamma(-s) \Gamma(1+s)} \tau^{-s} d s,-\min \{1 / 2, \alpha\}<\gamma<0
$$

can be obtained.

Because the auxiliary function $L_{\alpha}$ is defined in Equation (20) as an inverse Mellin transform, its Mellin transform is given by the formula

$$
L_{\alpha}^{*}(s)=\int_{0}^{\infty} L_{\alpha}(\tau) \tau^{s-1} d \tau=\frac{1}{\alpha \sqrt{\pi}} \frac{\Gamma\left(\frac{1}{2}+s\right) \Gamma\left(-\frac{s}{\alpha}\right) \Gamma\left(1+\frac{s}{\alpha}\right)}{\Gamma(-s) \Gamma(1+s)},-\min \{1 / 2, \alpha\}<\Re(s)<0 .
$$

Now we derive a series representation of the fundamental solution $G_{\alpha}$ by employing the Formula (16) and the general theory of the Mellin-Barnes integrals ([19]). To arrive at a series representation, the contour of integration in the integral at the right-hand side of (16) has to be transformed to the infinite loop $\mathcal{L}_{+\infty}$ starting and ending at $+\infty$ and encircling all poles $s_{k}=1 / 2+k, k=0,1,2, \ldots$ of the gamma function $\Gamma\left(\frac{1}{2}-s\right)$ and all poles $s_{k}=\alpha+\alpha k, k=0,1,2, \ldots$ of the gamma function $\Gamma\left(1-\frac{s}{\alpha}\right)$.

For the sake of simplicity let us restrict ourselves to the case of the simple poles, i.e., we suppose that the conditions $1 / 2+k \neq \alpha+\alpha n$ are fulfilled for all $k, n \in N$, i.e., that the derivative order $\alpha$ cannot be represented in the form $\alpha=\frac{1 / 2+k}{1+n}, k, n \in \mathbb{N}$. In particular, evidently it is the case if $\alpha$ is not a rational number or a rational number in the form $\alpha=\frac{p}{2 q+1}, p, q \in \mathbb{N}$.

Taking into account the known formula

$$
\operatorname{res}_{s=-k} \Gamma(s)=\frac{(-1)^{k}}{k !}, k=0,1,2, \ldots,
$$

the Jordan lemma and the Cauchy residue theorem provide us with the desired series representation of $G_{\alpha}$ :

$$
G_{\alpha}(x, t)=\frac{1}{\alpha \sqrt{\pi}|x|}\left(\Sigma_{1}(y)+\Sigma_{2}(y)\right), y=\frac{4 t}{x^{2}},
$$

where

$$
\begin{gathered}
\Sigma_{1}(y)=\sum_{k=0}^{\infty} \frac{(-1)^{k}}{k !} \frac{\Gamma\left(\frac{\frac{1}{2}+k}{\alpha}\right) \Gamma\left(1-\frac{\frac{1}{2}+k}{\alpha}\right)}{\Gamma\left(\frac{1}{2}+k\right) \Gamma\left(1-\left(\frac{1}{2}+k\right)\right)} y^{-k-\frac{1}{2},} \\
\Sigma_{2}(y)=\alpha \sum_{k=0}^{\infty} \frac{(-1)^{k}}{k !} \frac{\Gamma\left(\frac{1}{2}-\alpha(k+1)\right) \Gamma(k+1)}{\Gamma(\alpha(k+1)) \Gamma(1-\alpha(k+1))} y^{-\alpha(k+1)} .
\end{gathered}
$$


Using the reflection formula for the gamma function and some elementary trigonometric formulas, the series for $\Sigma_{1}$ and $\Sigma_{2}$ can be represented in the following shorter form:

$$
\begin{gathered}
\Sigma_{1}(y)=y^{-\frac{1}{2}} \sum_{k=0}^{\infty} \frac{\sin (\pi(1 / 2+k))}{\sin (\pi(1 / 2+k) / \alpha))} \frac{(-y)^{-k}}{k !}=y^{-\frac{1}{2}} \sum_{k=0}^{\infty} \frac{1}{\sin (\pi(1 / 2+k) / \alpha))} \frac{y^{-k}}{k !} \\
\Sigma_{2}(y)=\alpha y^{-\alpha} \sum_{k=0}^{\infty} \frac{\tan (\pi \alpha(k+1))}{\Gamma(1 / 2+\alpha(k+1))}\left(-y^{\alpha}\right)^{-k} .
\end{gathered}
$$

In the case $\alpha=1$, we get

$$
\Sigma_{1}(y)=y^{-\frac{1}{2}} \sum_{k=0}^{\infty} \frac{(y)^{-k}}{(-1)^{k} k !}=y^{-\frac{1}{2}} \exp (-1 / y), \quad \Sigma_{2}(y) \equiv 0
$$

and thus the well-known formula

$$
G_{1}(x, t)=\frac{1}{\sqrt{\pi}|x|}\left(\Sigma_{1}(y)+\Sigma_{2}(y)\right)=\frac{1}{\sqrt{\pi}|x|} y^{-\frac{1}{2}} \exp (-1 / y)=\frac{1}{\sqrt{4 \pi t}} \exp \left(-\frac{x^{2}}{4 t}\right)
$$

for the fundamental solution of the one-dimensional diffusion equation.

Let us now introduce another auxiliary variable, namely, $z=\frac{1}{y}=\frac{x^{2}}{4 t}$. Then the formulas from above can be rewritten in the following form:

$$
G_{\alpha}(x, t)=\frac{1}{\alpha \sqrt{\pi}|x|}\left(\Sigma_{3}(z)+\Sigma_{4}(z)\right), z=\frac{x^{2}}{4 t}
$$

where

$$
\begin{gathered}
\Sigma_{3}(z)=z^{\frac{1}{2}} \sum_{k=0}^{\infty} \frac{1}{\sin (\pi(1 / 2+k) / \alpha))} \frac{z^{k}}{k !}, \\
\Sigma_{4}(z)=\alpha z^{\alpha} \sum_{k=0}^{\infty} \frac{\tan (\pi \alpha(k+1))}{\Gamma(1 / 2+\alpha(k+1))}\left(-z^{\alpha}\right)^{k} .
\end{gathered}
$$

It follows from the last formula that the asymptotic behavior of $G_{\alpha}$ as $z \rightarrow 0$ (e.g., as $x \rightarrow 0$ with a fixed $t>0$ or $t \rightarrow+\infty$ with a fixed $x \neq 0$ ) is of a power law type:

$$
G_{\alpha}(x, t) \approx \frac{1}{\alpha \sqrt{4 \pi} \sin \left(\frac{\pi}{2 \alpha}\right)} t^{-\frac{1}{2}}+\frac{\tan (\pi \alpha)}{4^{\alpha} \sqrt{\pi} \Gamma(1 / 2+\alpha)} t^{-\alpha}|x|^{2 \alpha-1} .
$$

We remember the readers that all derivations above are valid only under the condition $\alpha>1 / 2$ that we assumed to hold true.

To get the asymptotic behavior of the fundamental solution $G_{\alpha}$ as $z \rightarrow+\infty$ (e.g., as $|x| \rightarrow+\infty$ with a fixed $t>0$ or $t \rightarrow 0$ with a fixed $x \neq 0$ ) we again employ the Mellin-Barnes representation (16). This time, the contour of integration in the integral at the right-hand side of (16) has to be transformed to the infinite loop $\mathcal{L}_{-\infty}$ starting and ending at $-\infty$ and encircling all poles $s_{k}=-\alpha k, k=0,1,2, \ldots$ of the gamma function $\Gamma\left(\frac{s}{\alpha}\right)$.

In doing so we first get an asymptotic series

$$
G_{\alpha}(x, t) \approx-\frac{1}{\pi^{\frac{3}{2}}|x|} \sum_{k=1}^{\infty} \Gamma\left(\frac{1}{2}+\alpha k\right) \sin (\pi \alpha k)\left(-\left(\frac{1}{z}\right)^{\alpha}\right)^{k}, z \rightarrow+\infty
$$


and then the asymptotic formula

$$
G_{\alpha}(x, t) \approx \frac{\Gamma\left(\frac{1}{2}+\alpha\right) \sin (\pi \alpha)}{4^{-\alpha} \pi^{\frac{3}{2}}} t^{\alpha}|x|^{-2 \alpha-1}, \frac{x^{2}}{4 t} \rightarrow \infty .
$$

\section{Entropy Production Rate of the Alpha-Fractional Diffusion Process}

For $\alpha=1$, the fundamental solution $G_{\alpha}$ is a Gaussian probability density function evolving in time:

$$
G_{1}(x, t)=\frac{1}{\sqrt{4 \pi t}} \exp \left(-\frac{x^{2}}{4 t}\right) .
$$

As has been shown in [11], the fundamental solution $G_{\alpha}$ to the $\alpha$-fractional diffusion Equation (1) can be interpreted as a probability density function evolving in time for all values of $\alpha$ between 0 and 1, too. Let us mention that it follows from the asymptotic Formula (25) that the second spatial moment of the probability density function $G_{\alpha}$ does not exist and the mean squared displacement of the diffusing particles in the framework of the diffusion process that is governed by the $\alpha$-fractional diffusion Equation (1) is not finite. Thus the $\alpha$-fractional diffusion Equation (1) describes a kind of an anomalous diffusion. Still, we show in this section that the entropy production rate of a diffusion process that is governed by the $\alpha$-fractional diffusion Equation (1) is exactly the same as the one of the conventional diffusion process.

Let us start with some definitions and examples. In the case of a one-dimensional continuous random variable with the probability density function $p(x), x \in \mathcal{X} \subseteq \mathbb{R}$, we adopt the Shannon definition of the entropy:

$$
S(p)=-k \int_{-\infty}^{\infty} p(x) \ln (p(x)) d x,
$$

where the constant $k$ can be set to be equal to one without loss of the generality. The Shannon entropy (26) is a special case of the more general definitions by Mathai, Tsallis or Rényi and these entropies will be considered elsewhere.

Let us mention that the Shannon entropy of a Gaussian random variable defined by the probability density function

$$
\mathcal{N}\left(\mu ; \sigma^{2}\right)=\frac{1}{\sqrt{2 \pi \sigma^{2}}} \exp \left(-\frac{(x-\mu)^{2}}{2 \sigma^{2}}\right)
$$

has the form

$$
S\left(\mathcal{N}\left(\mu ; \sigma^{2}\right)\right)=\frac{1}{2}\left(1+\ln \left(2 \pi \sigma^{2}\right)\right) .
$$

Thus the entropy increases with the width $\sigma^{2}$ of the probability density function $\mathcal{N}\left(\mu ; \sigma^{2}\right)$, i.e., the broader the distribution (uncertainty of the event), the larger the entropy, so that the Shannon entropy can be interpreted as a measure of uncertainty of an event that is governed by a probability density function $p(x)$.

When a probability density function is time-dependent, the entropy (26) depends on time, too:

$$
S(p, t)=-\int_{-\infty}^{\infty} p(x, t) \ln (p(x, t)) d x
$$

For such time-dependent random processes, the entropy production rate $R$ defined by

$$
R(p, t)=\frac{d}{d t} S(p, t)
$$

is a very important characteristic that can be interpreted as a natural measure of the irreversibility of a process. Say, in the case of a diffusion process that is described by the one-dimensional diffusion 
equation with the diffusion coefficient taken to be equal to one and that is therefore governed by the Gaussian distribution $\mathcal{N}(0 ; 2 t)$, the Formula (27) leads to the following result:

$$
R(\mathcal{N}(0 ; 2 t))=\frac{d}{d t} S(\mathcal{N}(0 ; 2 t))=\frac{1}{2 t^{\prime}}
$$

i.e., the entropy production rate is strictly positive for $t>0$ and the diffusion process can be classified as an irreversible process.

Otherwise, a wave propagation described by the wave equation is a reversible process with the entropy production rate equal to zero for $t>0$.

It is worth mentioning that the entropy production rates for the time- and the space-fractional diffusion equations that were calculated in [3] and [5], respectively, depend on the derivative order $\alpha$ and increase with increasing of $\alpha$ from 1 (diffusion) to 2 (wave propagation) that results in the so called entropy production paradox (see $[3,5]$ for attempts of resolving this paradox).

To calculate the entropy of the process governed by the one-dimensional $\alpha$-fractional diffusion Equation (1), let us employ the representation (19) of its fundamental solution in terms of the auxiliary function $L_{\alpha}$. Substituting (19) into (28) and after some elementary transformations, we get the following chain of equalities:

$$
\begin{gathered}
S(\alpha, t)=-\int_{-\infty}^{\infty} \frac{1}{|x|} L_{\alpha}\left(\frac{x^{2}}{4 t}\right) \ln \left(\frac{1}{|x|} L_{\alpha}\left(\frac{x^{2}}{4 t}\right)\right) d x= \\
-\int_{0}^{\infty} \frac{2}{x} L_{\alpha}\left(\frac{x^{2}}{4 t}\right) \ln \left(\frac{1}{x} L_{\alpha}\left(\frac{x^{2}}{4 t}\right)\right) d x=-\int_{0}^{\infty} \frac{1}{\tau} L_{\alpha}(\tau) \ln \left(\frac{1}{2} t^{-\frac{1}{2}} \tau^{-\frac{1}{2}} L_{\alpha}(\tau)\right) d \tau= \\
-\int_{0}^{\infty} \frac{1}{\tau} L_{\alpha}(\tau)\left(-\frac{1}{2} \ln (t)+\ln \left(\frac{1}{2} \tau^{-\frac{1}{2}} L_{\alpha}(\tau)\right)\right) d \tau=A_{\alpha} \ln (t)+B_{\alpha},
\end{gathered}
$$

where

$$
A_{\alpha}=\frac{1}{2} \int_{0}^{\infty} L_{\alpha}(\tau) \tau^{-1} d \tau, \quad B_{\alpha}=-\int_{0}^{\infty} \frac{L_{\alpha}(\tau)}{\tau} \ln \left(\frac{1}{2} \tau^{-\frac{1}{2}} L_{\alpha}(\tau)\right) d \tau
$$

To determine the entropy production rate of $G_{\alpha}$, the constant $A_{\alpha}$ is determined in explicit form. The integral that defines $A_{\alpha}$ can be interpreted as the Mellin transform of the auxiliary function $L_{\alpha}$ at the point $s=0$. Because the Formula (21) for the Mellin transform of $L_{\alpha}$ was derived under the condition $-\min \{1 / 2, \alpha\}<\Re(s)<0$, the Mellin transform of $L_{\alpha}$ at the point $s=0$ will be calculated as the limit of the right-hand side of (21) as $s \rightarrow 0$. Thus we get the following chain of equalities:

$$
\begin{aligned}
A_{\alpha}= & \frac{1}{2} \int_{0}^{\infty} L_{\alpha}(\tau) \tau^{-1} d \tau=\frac{1}{2} \lim _{s \rightarrow 0} \frac{1}{\alpha \sqrt{\pi}} \frac{\Gamma\left(\frac{1}{2}+s\right) \Gamma\left(-\frac{s}{\alpha}\right) \Gamma\left(1+\frac{s}{\alpha}\right)}{\Gamma(-s) \Gamma(1+s)}= \\
& \frac{1}{2 \alpha \sqrt{\pi}} \lim _{s \rightarrow 0} \Gamma\left(\frac{1}{2}+s\right) \frac{\sin (\pi s)}{\sin (\pi s / \alpha)}=\frac{\Gamma\left(\frac{1}{2}\right)}{2 \alpha \sqrt{\pi}} \lim _{s \rightarrow 0} \frac{\pi s}{\pi s / \alpha}=\frac{1}{2} .
\end{aligned}
$$

This formula along with the Formula (30) leads to the following expression for the entropy production rate $R(t)$ of the $\alpha$-fractional diffusion process described by the Equation (1):

$$
R(t)=\frac{d}{d t} S(\alpha, t)=\frac{1}{2 t} .
$$

This formula shows that $R(t)$ does not depend on the equation order $\alpha$ and is exactly the same as the entropy production rate of the conventional one-dimensional diffusion equation.

\section{Conclusions}

In this paper, a special case of the time- and space-fractional diffusion equation has been considered, namely, the case when the quotient of the orders of the time- and space-fractional 
derivatives is equal to one half as it is the case in the conventional diffusion equation. This special choice of the derivative orders leads to several important consequences. On the one hand, the fundamental solution to the $\alpha$-fractional equation can be expressed via an auxiliary function of the $\operatorname{argument} \frac{x^{2}}{4 t}$ like in the case of the conventional diffusion equation. Another important property of the $\alpha$-fractional diffusion equation is that its entropy production rate is exactly the same as the one of the diffusion process. Thus the $\alpha$-fractional diffusion equation can be considered to be a "natural fractionalization" of the diffusion equation. On the other hand, the mean squared displacement of the diffusing particles governed by the $\alpha$-fractional diffusion equation is infinite and thus this equation describes an anomalous diffusion process.

Conflicts of Interest: The author declares no conflict of interest.

\section{References}

1. Metzler, R.; Jeon, J.-H.; Cherstvy, A.G.; Barkai, E. Anomalous diffusion models and their properties: Non-stationarity, non-ergodicity, and ageing at the centenary of single particle tracking. Phys. Chem. Chem. Phys. 2014, 16, 24128-24164.

2. Shannon, C.E. A mathematical theory of communication. Bell Syst. Tech. J. 1948, 27, 379-423.

3. Hoffmann, K.H.; Essex, C.; Schulzky, C. Fractional diffusion and entropy production. J. Non-Equilib. Thermodyn. 1998, 23, 166-175.

4. Li, X.; Essex, C.; Davison, M.; Hoffmann, K.H.; Schulzky, C. Fractional diffusion, irreversibility and entropy. J. Non-Equilib. Thermodyn. 2003, 28, 279-291.

5. Prehl, J.; Essex, C.; Hoffmann, K.H. The superdiffusion entropy production paradox in the space-fractional case for extended entropies. Phy. A 2010, 389, 214-224.

6. Prehl, J.; Essex, C.; Hoffmann, K.H. Tsallis relative entropy and anomalous diffusion. Entropy 2012, 14, 701-716.

7. Luchko, Y. Wave-diffusion dualism of the neutral-fractional processes. J. Comput. Phys. 2015, 293, 40-52.

8. Mathai, A.M.; Rathie, P.N. Basic Concepts in Information Theory and Statistics: Axiomatic Foundations and Applications; Wiley Eastern: New Delhi, India, 1975.

9. Mathai, A.M.; Haubold, H.J. Pathway model, superstatistics, Tsallis statistics and a generalized measure of entropy. Phys. A 2007, 375, 110-122.

10. Mathai, A.M.; Haubold, H.J. On generalized entropy measures and pathways. Phys. A 2007, 385, 493-500.

11. Mainardi, F.; Luchko, Yu.; Pagnini, G. The fundamental solution of the space-time fractional diffusion equation. Fract. Calc. Appl. Anal. 2001, 4, 153-192.

12. Gorenflo, R.; Mainardi, F. Random walk models approximating symmetric space-fractional diffusion processes. In Problems in Mathematical Physics; Elschner, J., Gohberg, I., Silbermann, B., Eds.; Birkhäuser Verlag: Basel, Switzerland, 2001; pp. 120-145.

13. Saichev, A.; Zaslavsky, G. Fractional kinetic equations: Solutions and applications. Chaos 1997, 7, 753-764.

14. Samko, S.G.; Kilbas, A.A.; Marichev, O.I. Fractional Integrals and Derivatives: Theory and Applications; Gordon and Breach: New York, NY, USA, 1993.

15. Feller, W. On a generalization of Marcel Riesz' potentials and the semi-groups generated by them. Comm. Sém. Mathém. Univ. Lund. 1952, 73-81.

16. Gorenflo, R.; Mainardi, F. Random walk models for space-fractional diffusion processes. Frac. Calc. Appl. Anal. 1998, 1, 167-191.

17. Luchko, Y. Operational method in fractional calculus. Fract. Calc. Appl. Anal. 1999, 2, 463-489.

18. Luchko, Y.; Gorenflo, R. An operational method for solving fractional differential equations with the Caputo derivatives. Acta Math. Vietnam. 1999, 24, 207-233.

19. Marichev, O.I. Handbook of Integral Transforms of Higher Transcendental Functions, Theory and Algorithmic Tables; Ellis Horwood: Chichester, UK, 1983. 
20. Luchko, Yu.; Kiryakova, V. The Mellin integral transform in fractional calculus. Fract. Calc. Appl. Anal. 2013, $16,405-430$.

(C) 2016 by the author; licensee MDPI, Basel, Switzerland. This article is an open access article distributed under the terms and conditions of the Creative Commons by Attribution (CC-BY) license (http://creativecommons.org/licenses/by/4.0/). 